\title{
A benchmark for particle shape dependence
}

\author{
Gaël Combe*, Cécile Nouguier-Lehon ${ }^{\dagger}$, Émilien Azéma**, Krzysztof Szarf*, \\ Baptiste Saint-Cyr**, Marie Chaze ${ }^{\dagger}$, Farhang Radjai**, Pascal Villard*, Jean-Yves \\ Delenne*, Vincent Richefeu*, Philippe Sornay ${ }^{\S}$, Charles Voivret ${ }^{\mathbb{I l}}$ and CEGEO \\ ${ }^{*}$ UJF-Grenoble 1, Grenoble-INP, CNRS UMR 5521, 3SR Lab., Grenoble, France. \\ ${ }^{\dagger}$ University of Lyon, Ecole Centrale de Lyon, LTDS UMR CNRS 5513, Ecully, France. \\ ${ }^{* *}$ University Montpellier 2, CNRS, LMGC UMR 5508, Montpellier, France. \\ ${ }^{\ddagger}$ IATE, UMR 1208 INRA-CIRAD-Montpellier Supagro-UM2, Montpellier, France. \\ ${ }^{\S} C E A, D E N$, SPUA, LCU, Saint Paul lez Durance, France. \\ "SNCF Innovation and Research, Paris, France. \\ "Collaborative group "Changement d'Échelle dans les GÉOmatériaux" (scale change in geomaterials).
}

\begin{abstract}
Particle shape is a major parameter for the space-filling and strength properties of granular materials. For a systematic investigation of shape effect, a numerical benchmark test was set up within a collaborative group using different numerical methods and particles of various shape characteristics such as elongation, angularity and nonconvexity. Extensive 2D shear simulations were performed in this framework and the shear strength and packing fraction were compared for different shapes. We show that the results may be analyzed in terms of a low-order shape parameter $\eta$ describing the degree of distortion from a perfectly circular shape. In particular, the shear strength is an increasing function of $\eta$ with nearly the same trend for all shapes, the differences being of second order compared to $\eta$. We also observe a nontrivial behavior of packing fraction which, for all our simulated shapes, increases with $\eta$ from the random close packing fraction for disks, reaches a peak considerably higher than that for disks, and subsequently declines as $\eta$ is further increased. Finally, the analysis of contact forces for the same value of $\eta$ leads to very similar statistics regardless of our specific particle shapes.
\end{abstract}

Keywords: Granular Media, Clumps, Polygones, Molecular Dynamic, Contact Dynamic, Shape Dependence, Shear Test. PACS: 45.70.-n, 81.05.Rm, 61.43.Hv

\section{INTRODUCTION}

Particle shape is a major parameter for the spacefilling and strength properties of matter including liquids, glasses and granular materials [1,2]. Since the experiments of Oda [3] performed on assemblies of oval cross-sectional rods, several studies have been reported and rather recent results suggest that assemblies of nonspherical particles exhibit higher shear strength than sphere packings and may approach unusually high packing fractions $[2,4,5,6]$. However, such results are often limited to a specific shape parameter, such as angularity with polygonal particles [7, 8, 9], elongation with ellipsoids or elongated faceted particles $[10,11,12,13,14$, $15]$, or nonconvexity $[16,17,18]$. Moreover the effect of each of these parameters is experimentally difficult to investigate in a systematic way.

For a systematic investigation of shape effect, a 2D numerical benchmark test was performed within the collab-

http://www.cgp-gateway.org/ orative group CEGEO using different numerical methods and particles of various shape characteristics. In a first section, the definition of a low-order shape parameter is given to characterize the degree of distortion from a perfectly spherical or circular shape. Then the numerical benchmark is presented and the results are analyzed in terms of this shape parameter. We present especially the evolution of shear strength, packing fraction and contact force distributions.

\section{$\eta$, A PARTICLE SHAPE PARAMETER}

We define the $\eta$-set as the set of all shapes with borders enclosed between a pair of concentric circles (spheres in 3D), touching both circles and having the same ratio $\eta=\Delta R / R$. Figure 1 shows examples of four different particle shapes belonging to the same $\eta$-set. The parameter $\eta$ is obviously a rough low-order shape parameter, but allowing to deal with very different particle shapes as the main purpose of our benchmark. For instance, the A-shape (trimer) is composed of three overlapping disks touching the circumscribing circle and with their intersection points lying on the inscribed circle; the B-shape (rounded-cap rectangle) is a rectangle touching the in- 


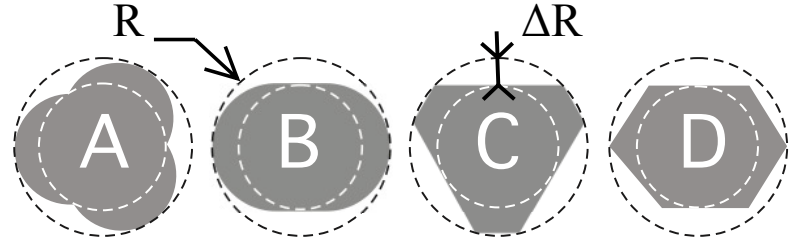

FIGURE 1. Four different shapes belonging to the same $\eta$ set with $\eta=\Delta R / R=0.4$ : trimer (A), rounded-cap rectangle $(B)$, truncated triangle $(C)$, elongated hexagon (D).

scribed circle and juxtaposed with two half-disks touching the circumscribing circle; the $\mathrm{C}$-shape (truncated triangle) is a hexagon with three sides constrained to touch the inscribed circle and all corners on the circumscribing circle; and the D-shape (elongated hexagon) is an irregular hexagon with two sides constrained to touch the inscribed circle and two corners lie on the circumscribing circle. The parameter $\eta$ describes the degree of distortion from a perfectly circular shape. In the case of shapes of figure 1 , the larger is $\eta$, the more concave is a particle of A-shape, the more elongated is a particle of B-shape and the more angular is a particle of C-shape. In the case of D-shape, a non-zero value of $\eta$ corresponds to a combination of elongation and angularity. The range of geometrically defined values of $\eta$ for a given shape (defined by a construction method) has in general a lower bound $\eta_{0}$. For $\mathrm{A}$ and $\mathrm{B}$, the particle shape changes continuously from a disk $(\Delta R=0)$, so that $\eta_{0}=0$ whereas for $\mathrm{C}$ and $\mathrm{D}$, because of their polygonal nature, their common lower bound is $\eta_{0}=1-\sqrt{3} / 2 \simeq 0.13$, a regular hexagon.

\section{BENCHMARK}

The benchmark test is a 2D shear simulation with specified dimensionless parameters. For each shape of Fig. 1, several packings of 5000 particles were prepared with $\eta$ varying from $\eta_{0}$ to 0.5 . To avoid long-range ordering, a size polydispersity was introduced by taking $R$ in the range $\left[R_{\min }, R_{\max }\right]$ with $R_{\max }=3 R_{\min }$ and a uniform distribution of particle volumes [19]. A dense packing composed of disks $(\eta=0)$ was first constructed by means of random deposition in a box. For other values of $\eta$, the same packing was used with each disk serving as the circumscribing circle. The particle was inscribed with the desired value of $\eta$ and random orientation inside the disk. This geometrical step was followed by isotropic compaction of the packings inside a rectangular frame. The gravity $g$ and friction coefficients between particles and with the walls were set to 0 during compaction in order to avoid force gradients. Figure 2 displays snapshots of the packings for $\eta=0.4$ at the end of isotropic compaction. Then the isotropic samples were sheared by applying a slow downward velocity on the top wall with a constant confining stress $p_{0}$ acting on the lateral walls (the inertia parameter $I$ defined in $[20,21]$ is kept below $10^{-3}$ ). During shear, the friction coefficient $\mu$ between particles was set to 0.5 and to 0 with the walls.
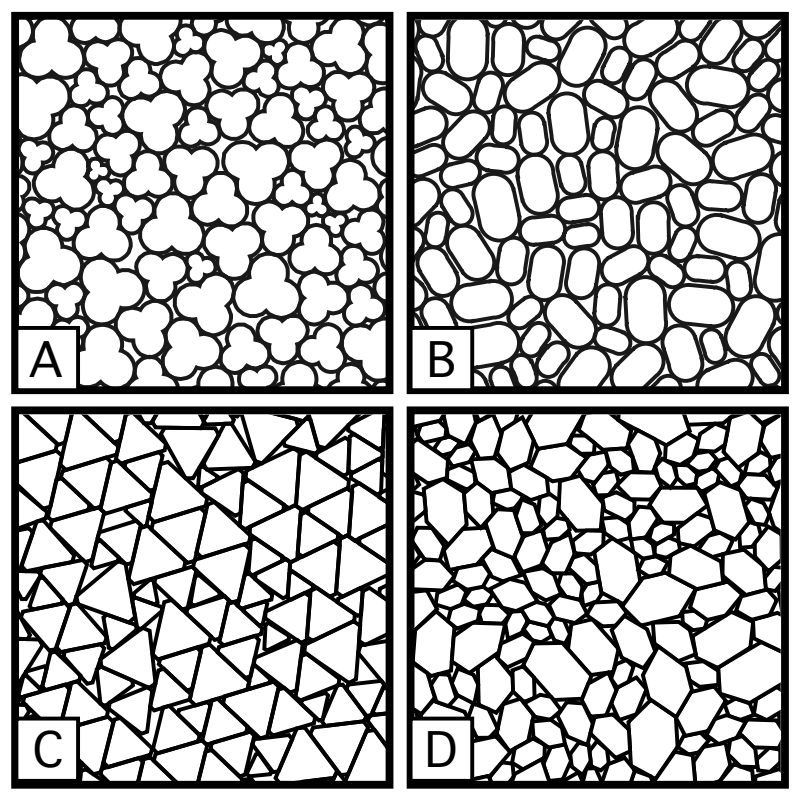

FIGURE 2. Snapshots of the simulated packings in the densest isotropic state.

\section{Granular models}

Two different discrete element methods (DEM) were used for the simulations: contact dynamics (CD) and molecular dynamics (MD). In the CD method, the particles are treated as perfectly rigid [22] whereas a linear spring-dashpot model was used in MD simulations with stiff particles $\left(k_{n} / p_{0}=10^{3}\right.$, where $k_{n}$ is the normal stiffness and $p_{0}$ refers to the confining pressure [20]). The trimers were simulated by both methods for all values of $\eta$. We refer below as packings A and A' (for CD and MD methods, respectively) to these simulations. The packing $\mathrm{C}$ was simulated by MD whereas the packings $\mathrm{B}$ and $\mathrm{D}$ were simulated by $\mathrm{CD}$.

\section{Shear strength dependence with $\eta$}

The shear strength is characterized by the internal angle of friction $\varphi$ defined by $\sin \varphi=\frac{\sigma_{1}-\sigma_{2}}{\sigma_{1}+\sigma_{2}}$, where the

Animation videos of the simulations can be found at www.cgp-
gateway.org/ref012.html 
subscripts 1 and 2 refer to the principal stresses. Since the samples are dense, $\sin \varphi$ increases rapidly from zero to a peak value before relaxing to a constant materialdependent value $\sin \varphi^{*}$, which defines the shear strength at large strain at a steady stress state.

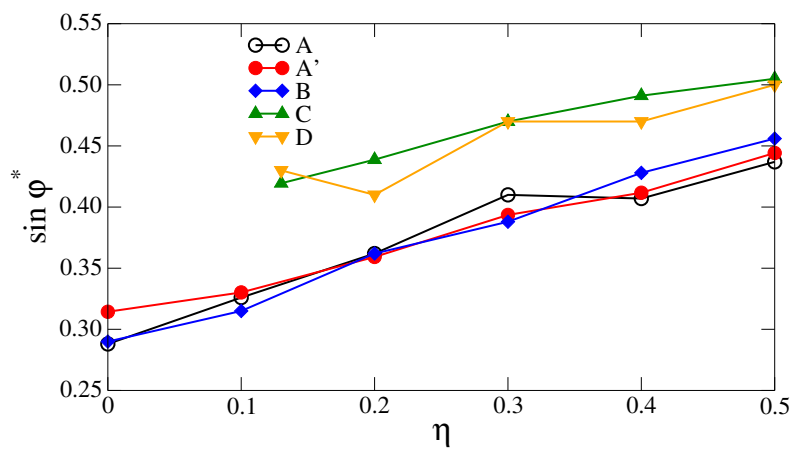

FIGURE 3. Shear strength $\sin \varphi^{*}$ of packings composed of various particle shapes as a function of $\eta$.

Figure 3 shows the dependence of $\sin \varphi^{*}$ with respect to $\eta$ for our different shapes. Remarkably, $\sin \varphi^{*}$ increases with $\eta$ at the same rate for all shapes. The data nearly coincide between the A and B shapes, on the one hand, and between $\mathrm{C}$ and $\mathrm{D}$ shapes, on the other hand. This suggests that nonconvex trimers and rounded-cap rectangles, in spite of their very different shapes, belong to the same family (rounded shapes). In the same way, the truncated triangles and elongated hexagons seem to belong to the family of angular particles and exhibit a shear strength slightly above that of rounded shapes. Note also that the results are robust with respect to the numerical approach as the packings A and A' were simulated by two different numerical methods.

The increase of shear strength with $\eta$ may be attributed to the increasing frustration of particle rotations as the shape deviates from disk. Since the particles may interact at two or three contact points (A-shape) or through side-to-side contacts (shapes B, C and D), the kinematic constraints increase with $\eta$ and frustrate the particle displacements by rolling. The restriction of rolling leads to enhanced role of friction in the mechanical equilibrium and relative sliding of particles during deformation [23]. A related static quantity is the mean friction mobilization defined by $M=\left\langle f_{t} /\left(\mu f_{n}\right)\right\rangle$, where $f_{t}$ is the magnitude of the friction force, $f_{n}$ is the normal force, and the average is taken over all force-bearing contacts in the system.

To evaluate the effect of particle shape, we consider the parameter $M_{\eta}=M(\eta) / M(0)-1$ as a function of $\eta$ for different shapes, where $M(0)$ is the friction mobilization for circular particles. Figure 4 shows that $M_{\eta}$ is a globally increasing function of $\eta$ for all shapes. The parameter $\eta$ appears also in this respect to account for the global trend of friction mobilization, and the differences

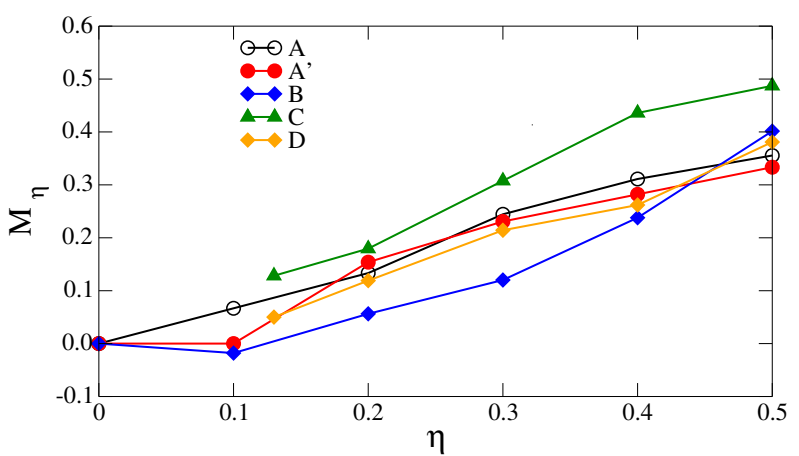

FIGURE 4. Friction mobilization in the steady state as a function of $\eta$ for different particle shapes.

observed in Fig. 4 among different shapes are rather of second order.

\section{Packing fraction dependence with $\eta$}

The packing fraction for samples in the initial isotropic state was fully studied in [24]. It was observed a nontrivial behavior for all particle shapes: the packing fraction increases with $\eta$ from the random close packing fraction for disks, reaches a peak considerably higher than that for disks, and subsequently declines as $\eta$ is further increased. For the B-shape a sharp decrease of $\rho^{\text {iso }}$ occurs beyond $\eta=0.5$ as was shown in [13]. This unmonotonic behavior of packing fraction is an agreement with the literature [24]. The decrease of the packing fraction was attributed to the excluded-volume effect that prevails at large aspect ratios and leads to increasingly larger pores which cannot be filled by the particles [25]. The observation of this unmonotonic behavior as a function of $\eta$ for different shapes indicates that it is a generic property depending only on deviation from circular shape. The analytical model of $\rho^{\text {iso }}$ suggested in [24] has shown that the packing fraction is rules by both $\eta$ and a second-order parameter corresponding to the selfporosity of each grain shape.

\section{Contact force dependence with $\eta$}

The number of contacts between two grains depends more on the shape than on $\eta$, provided that $\eta$ is different from extreme values, e.g., 0 for A and A' shapes, 0.5 for $C$ shapes, etc. For $C$ and D shapes, one or two contact points are observed between two particles (respectively face-corner and face-face contacts [14, 18]). For $A-A^{\prime}$ and $B$ shapes, one to four contact points are possible $[13,17,18]$. In each of those contacts, a normal force is computed. More than the individual values of the con- 
tact forces, the level of the normal force between two grains is also an interesting information. Figure 5 shows the statistical distribution of $f_{n}$, where $f_{n}$ is the sum over $1,2,3$ or 4 normal contact forces between each frictionless grains in dense packings submitted to an isotropic loading. A remarkable observation is that for all shapes the values of $f_{n}$ have a similar distribution, excepted for the $C$ shape for which the distribution is narrower (for $C$ shapes $\eta=0.5$ is an upper limit corresponding to an isosceles triangle shape; assembly of such shapes showing high proportions of face-corner contacts).

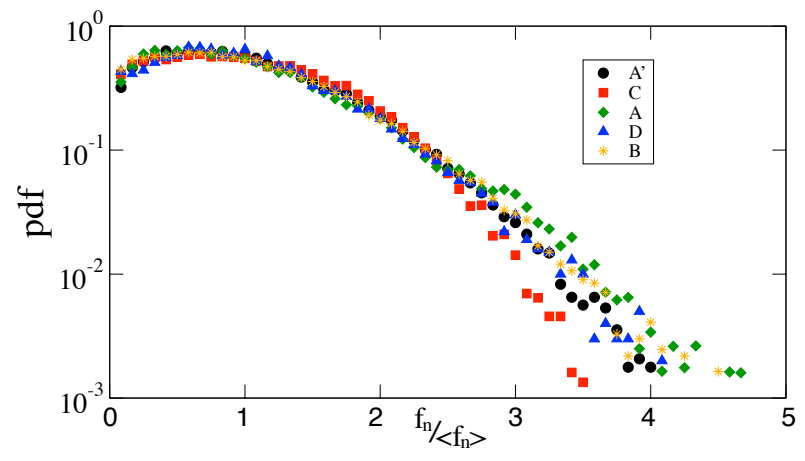

FIGURE 5. Probability density function (pdf) of normalized forces $f_{n} /\left\langle f_{n}\right\rangle$ between grains for each shapes, with $\eta=0.5$.

\section{CONCLUSIONS}

Our 2D benchmark simulations show that the packing fraction and shear strength of granular media composed of noncircular particles may be analyzed in terms of a low-order shape parameter $\eta$, describing deviation with respect to circular shape. The shear strength is an increasing function of $\eta$ with nearly the same trend for all our simulated shapes, the differences being of second order compare to $\eta$. For our simulated shapes, the packing fraction increases with $\eta$, passes by a peak depending on each specific shape and subsequently declines. Finally, the analysis of contact forces for the same value of $\eta$ leads to very similar statistics regardless of our specific particle shapes.

An interesting issue to be addressed in future is to explore the microscopic origins of the $\eta$-dependence exposed in this paper.

\section{ACKNOWLEDGMENTS}

We also acknowledge financial support of the French government through the program PPF CEGEO.

\section{REFERENCES}

1. K. Binder, W. Kob Glassy materials and disordered solids World Scientific (2005) 452.

2. W. Man, A. Donev, F. Stillinger, M. Sullivan, W. Russel, D. Heeger, S. Inati, S. Torquato, P. Chaikin Phys. Rev. Lett. 94 (2005) 198001.

3. M. Oda, J. Konisho, S. Nemat-Nasser Mechanics of Materials 1 (1982) 269.

4. A. Donev, F. Stillinger, P. Chaikin, S. Torquato Science 303 (2004) 990.

5. A. Donev, I. Cisse, D. Sachs, E. Variano, F. Stillinger, R. Connelly, S. Torquato, P. Chaikin Phys. Rev. Lett. 92 (2004) 255506.

6. Y. Jiao, F. Stillinger, S. Torquato Phys. Rev. E 1 (2010) 041304.

7. A. Mirghasemi, L. Rothenburg, E. Maryas Géotechnique 52 (2002) 209.

8. E. Azéma, F. Radjai, R. Peyroux, G. Saussine Phys. Rev. E 76 (2007) 011301.

9. E. Azéma, F. Radjai, G. Saussine Mechanics of Materials 41 (2009) 721.

10. T.-T Ng Int. J. Numer. Anal. Meth. Geomech 33 (2009) 511.

11. C. Nouguier-Lehon, B. Cambou, E. Vincens Int. J. Numer. Anal. Meth. Geomech 27 (2003) 27.

12. E.-M. Charalampidou, G. Combe, G. Viggiani, J. Lanier "Mechanical behavior of mixtures of circular and rectangular 2D particles", in Powders and Grains 2009 (2009) 821.

13. E. Azéma, F. Radjai Phys. Rev. E 81 (2010) 051304.

14. C. Nouguier-Lehon Comptes Rendus Mecanique 338 (2010) 587.

15. R. Hidalgo, I. Zuriguel, D. Maza, I. Pagonabarraga J. Stat. Mech. (2010) P06025.

16. K. Szarf, G. Combe, P. Villard "Influence of the grains shape on the mechanical behavior of granular materials", in Powders and Grains 2009 (2009) 357.

17. B. Saint-Cyr, J.-Y Delenne, C. Voivret, F. Radjai, P. Sornay Phys. Rev. E 84 (2011) 041302.

18. K. Szarf, G. Combe, P. Villard Powder Technology 208 (2011) 279.

19. C. Voivret, F. Radjai, J.-Y Delenne, M.S.E. Youssoufi Phys. Rev. E 76 (2007) 021301.

20. G. Combe, J.-N. Roux "Discrete numerical simulation, quasistatic deformation and the origins of strain in granular materials", in Deformation Characteristics of Geomaterials (2003) 1071.

21. GDR MiDi Eur. Phys. J. E 14 (2004) 341.

22. F. Radjai, V. Richefeu Mechanics of Materials 41 (2009) 715.

23. N. Estrada, A. Taboada, F. Radjai Phys. Rev. E 78 (2008) 021301.

24. CEGEO, B. Saint-Cyr, K. Szarf, C. Voivret, É. Azéma, V. Richefeu, J.-Y. Delenne, G. Combe, C. Nouguier-Lehon, P. Villard, P. Sornay, M. Chaze, F. Radjaï Europhysics Letters 98(4) (2012) 44008.

25. S. Williams, A. Philipse Phys. Rev. E 67 (2003) 051301. 